\title{
The Backscattering Correction Factor in AES: A New Outlook
}

\author{
A. Jablonski ${ }^{1 *}$ and C. J. Powell ${ }^{2}$ \\ ${ }^{1}$ Institute of Physical Chemistry, Polish Academy of Sciences, ul. \\ Kasprzaka 44/52, 01-224 Warsaw, Poland \\ ${ }^{2}$ Surface and Microanalysis Science Division, National Institute of Standards and Technology, \\ Gaithersburg, Maryland 20899-8370, USA \\ jablo@ichf.edu.pl
}

(Received: October 3, 2010; Accepted : January 6, 2011)

\begin{abstract}
There is currently renewed interest in the backscattering correction for Auger electron spectroscopy (AES). There are several reasons for this interest. First, the energy of the primary-beam energy reaches $25 \mathrm{keV}$ in modern AES instruments and Shimizu's predictive formulae based on calculations for primary energies between $3 \mathrm{keV}$ and $10 \mathrm{keV}$ are of uncertain validity at higher energies. Second, it has been shown recently that the present definition of the backscattering factor is based on a simplified model of electron transport that breaks down for low primary energies and/or more grazing-incidence angles. A new term, the backscattering correction factor (BCF) has therefore been introduced that is based on an advanced model of electron transport. Third, much progress has been recently made in the theory of electron transport and the data for electron-scattering parameters which should improve the reliability of calculated backscattering correction factors. Finally, the BCF from the advanced theoretical model has been found to depend on numerous parameters defining the solid, the selected Auger transition, and the experimental configuration. Since the derivation of a simple predictive formula for the BCF does not seem to be feasible, a computer-controlled database has been developed to provide BCFs for a user-specified material and experimental configuration. Examples are given of BCFs from the advanced and simplified models for Ag $\mathrm{M}_{4} \mathrm{~N}_{45} \mathrm{~N}_{45}$ Auger electrons from silver.
\end{abstract}

\section{Introduction}

The relation between the Auger electron signal intensity and the number of atoms of a given element in a solid is generally expressed in terms of a set of parameters similar to those used electron microprobe analysis. For an elemental solid, this relation typically has the form [1-3]

$$
I_{A}=C_{A} \lambda_{\text {in }} Q_{A} R N
$$

where $C_{A}$ is a parameter depending on instrumental configuration, particular settings, and the selected Auger transition, $\lambda_{\text {in }}$ is the inelastic mean free path of the detected Auger electrons, $Q_{A}$ is the correction for elastic scattering of the Auger electrons, $N$ is the atomic density of the element (number of atoms per unit volume), and $R$ is the backscattering factor (BF). The latter parameter accounts for additional ionizations of atoms in the surface region due to backscattered electrons. The correction for electron-backscattering effects was proposed by
Bishop and Riviere [4] in one of the first papers on the formalism for quantitative AES analysis.

Recent advances in the theory of electron transport in solids have led to the conclusion that the theoretical model assumed in previous derivations of the formula for the backscattering factor is oversimplified [5-11]. As a result, BFs calculated from this simplified model are unreliable for low primary energies and/or more-grazing incidence angles. Much effort has been devoted recently to the development of an advanced theoretical model that would accurately predict Auger-electron signal intensities for a wide range of primary energies and experimental configurations. It has also been found necessary to define a new term, the backscattering correction factor (BCF), to describe the correction needed for quantitative AES. Finally, the BCF has been found to depend on an additional parameter, the Auger-electron emission angle.

Four issues are briefly addressed in the present report. We describe the definition of the backscattering correction factor in Section 2 that is based on the advanced model of electron 
transport. It has been recently found that the $\mathrm{BCF}$ depends not only on the Auger-electron emission angle but also on the shape of the solid acceptance angle of the analyzer [11]. The relevant theory is summarized in Section 3, while an example illustrating this effect is given in Section 4. Finally, a brief description is given of a new National Institute of Standards and Technology (NIST) database that provides BCFs from the simplified and advanced theoretical models for a user-specified Auger transition and instrumental configuration.

\section{Definitions}

According to the ASTM International terminology standard, the backscattering factor is defined as "the fractional increase in the Auger current due to backscattered electrons" [12]. A similar definition of this parameter is found in the vocabulary standard of the International Organization for Standardization (ISO) [13]: "Factor defining the increase in the Auger electron current due to additional ionizations in the sample caused by backscattered electrons above that arising directly from the primary electrons". Both definitions indicate that the backscattering factor is larger than unity. There is some ambiguity in usage, however, with some authors referring to the $\mathrm{BF}$ as the fractional increase defined above (often denoted $r$ ) while other authors refer to the $\mathrm{BF}$ as the correction, $R$, of the AES signal intensity as in Eq. (1), where $R=1+r$. To clarify this situation, a note was added to the ISO definition: "Different usages exist; the factor is commonly the fractional increase, as defined above, and sometimes unity plus that fractional increase. The latter usage is deprecated. For clarity, the particular usage needs to be defined."

The ISO Technical Committee on Surface Chemical Analysis is currently considering the definitions of two new terms for AES: the backscattering correction factor (BCF) and the backscattering fraction. The BCF is defined as "equal to the ratio of the total Auger-electron current arising from ionizations in the sample caused by both the primary electrons and the backscattered electrons to the Auger-electron current arising directly from the primary electrons". The backscattering fraction is defined as "the ratio of the Auger-electron current arising from ionizations in the sample caused by backscattered electrons and the Auger-electron current arising directly from the primary electrons". Both definitions are accompanied by the same note: "In simple theories, evaluations of the backscattering correction factor or the backscattering fraction may be based on the assumption that the primary beam is unchanged, in intensity, energy or direction, within the information depth for Auger-electron emission. This assumption becomes progressively less useful as the primary energy becomes closer to the core-level ionization energy for the relevant Auger transition or for increasing angles of incidence of the primary electrons. In such cases, a more advanced theory of electron transport should be used. For example, if the primary energy is less than twice the core-level ionization energy, the total Auger-electron current emitted from the sample may be less than that calculated for an unaltered primary beam alone so that the effective value of the backscattering fraction is then negative. In addition, the separate classification of electrons as primary or backscattered becomes progressively less useful."

In the present work, we utilize only the first new term, the BCF.

\section{Theory}

Jablonski [5] has shown that the general formula for the $\mathrm{BCF}$, resulting from the advanced theoretical model, has the general form:

$$
R=\frac{\int_{\Delta \Omega} \int_{0}^{\infty} \Phi\left(z, E_{0}, \theta_{0}\right) \phi(\Omega, z) d z d \Omega}{\int_{\Delta \Omega} \int_{0}^{\infty} \Phi^{i d}\left(E_{0}, \theta_{0}\right) \phi(\Omega, z) d z d \Omega} .
$$

where $\Delta \Omega$ is the solid acceptance angle of the analyzer, $\Phi\left(z, E_{0}, \theta_{0}\right)$ is the excitation depth distribution function (EXDDF), $\Phi^{i d}\left(E_{0}, \theta_{0}\right)$ is the EXDDF for the idealized case of ionizations created only by primary electrons, and $\phi(\Omega, z)$ is the emission depth distribution function (EMDDF). The EXDDF is a function of depth $z$, primary energy $E_{0}$, and the primary-electron incidence angle $\theta_{0}$. The EMDDF is a function of the direction of emitted Auger electrons within the acceptance angle of the analyzer, $\Omega$, and the depth $z$. This notation is illustrated in Fig. 1 which shows an example of an experimental configuration with an analyzer having a solid angle corresponding to a cone with semi-angle $\Delta \alpha$ and with its axis perpendicular to the sample surface.

The EXDDF can be conveniently normalized with respect to the number of ionizations by the primary electrons alone: 


$$
\Phi^{N}\left(z, E_{0}, \theta_{0}\right)=\frac{\Phi\left(z, E_{0}, \theta_{0}\right)}{\Phi^{i d}\left(E_{0}, \theta_{0}\right)} .
$$

Suppose that a given direction $\Omega$ is located at an Auger-electron emission angle $\alpha$. One of the normalization conventions for the EMDDF is that the integral over depth is equal to unity [14]:

$$
\phi^{*}(\alpha, z)=\frac{\phi(\alpha, z)}{\int_{0}^{\infty} \phi(\alpha, z) d z} .
$$

Such a normalization leads to a convenient expression for the $\mathrm{BCF}$ for an analyzer with a small solid acceptance angle [5,11]:

$$
R=\int_{0}^{\infty} \Phi^{N}\left(z, E_{0}, \theta_{0}\right) \phi^{*}(\alpha, z) d z .
$$

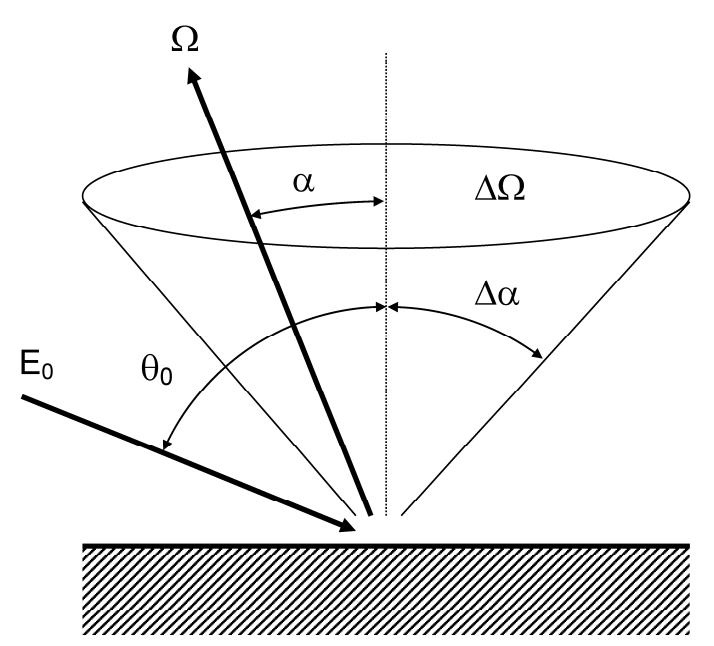

Fig. 1. Outline of an experimental AES configuration with an analyzer having a large conical acceptance angle.

Jablonski and Powell [11] analyzed the case of an analyzer with a large solid acceptance angle, $\Delta \Omega$. These authors introduced a new function, the integral emission depth distribution function (IEMDDF), to simplify the notation and BCF calculation. The following defining formula for the IEMDDF was proposed:

$$
\phi^{N}(\Delta \Omega, z)=\frac{\int_{\Delta \Omega} \phi(\Omega, z) d \Omega}{\int_{\Delta \Omega} \int_{0}^{\infty} \phi(\Omega, z) d z d \Omega} .
$$

Introducing Eqs. (3) and (6) into Eq. (2), we obtain [11],

$$
R=\int_{0}^{\infty} \Phi^{N}\left(z, E_{0}, \theta_{0}\right) \phi^{N}(\Delta \Omega, z) d z,
$$

to give the BCF from the advanced model.

Jablonski [5] has shown that, under certain assumptions, Eqs. (2) and (5) simplify to the form often used in previous calculations (i.e., the simplified model):

$$
\begin{aligned}
& R=1+ \\
& \frac{\cos \theta_{0}}{I_{0} \sigma_{i}\left(E_{0}\right)} \int_{E_{c}}^{E_{0}} \int_{0}^{\pi / 2} \frac{I_{B}\left(E, \alpha_{B}\right) \sigma_{i}(E)}{\cos \alpha_{B}} d \alpha_{B} d E,
\end{aligned}
$$

where $\sigma_{i}(E)$ is the relevant inner-shell ionization cross section at an electron energy $E$, $I_{0}$ is the primary-electron current, and $I_{B}\left(E, \alpha_{B}\right)$ is the energy and angular distribution of backscattered electrons. Note that Eq. (8) is independent of the size and location of the analyzer solid angle.

\section{Results and discussion}

We analyze the influence of the size of analyzer solid angle on the $\mathrm{BCF}$ for an illustrative Auger transition, $\mathrm{Ag} \mathrm{M}_{4} \mathrm{~N}_{45} \mathrm{~N}_{45}$ Auger electrons from silver. Calculations were performed for the experimental configuration shown in Fig. 1. The algorithm used for these calculations is described in Ref. 11. Figure 2 shows the IEMDDF calculated for two analyzer acceptance solid angles: a cone with $\Delta \alpha=10^{\circ}$ and a cone with $\Delta \alpha=80^{\circ}$. Although the analyzer axis is

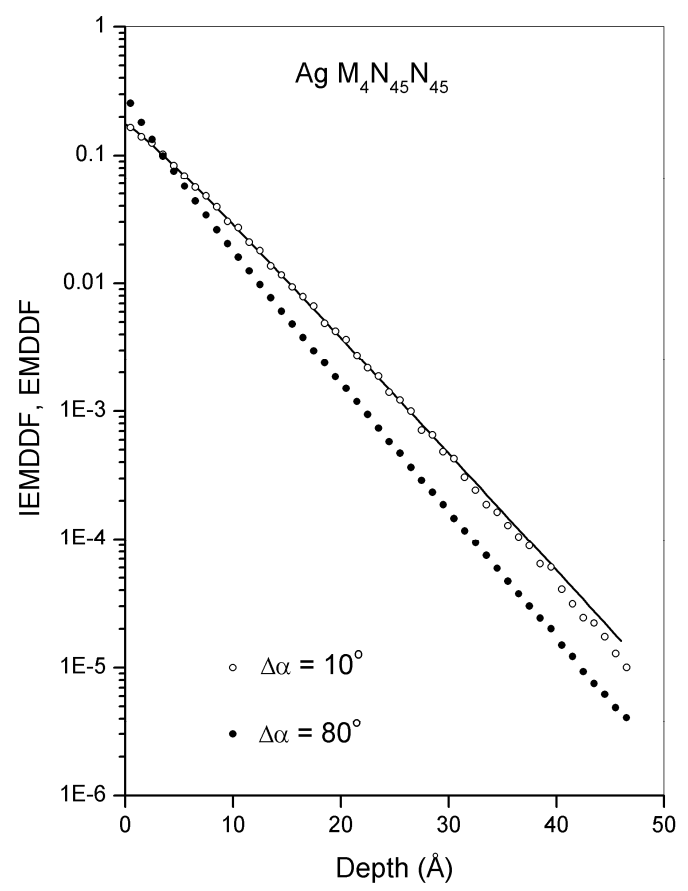

Fig. 2. The IEMDDF for $A g \mathrm{M}_{4} \mathrm{~N}_{45} \mathrm{~N}_{45}$ Auger electrons from silver. Open circles: $\Delta \alpha=10^{\circ}$; filled circles: $\Delta \alpha=80^{\circ}$; solid line: the EMDDF calculated from the transport approximation [15]. 

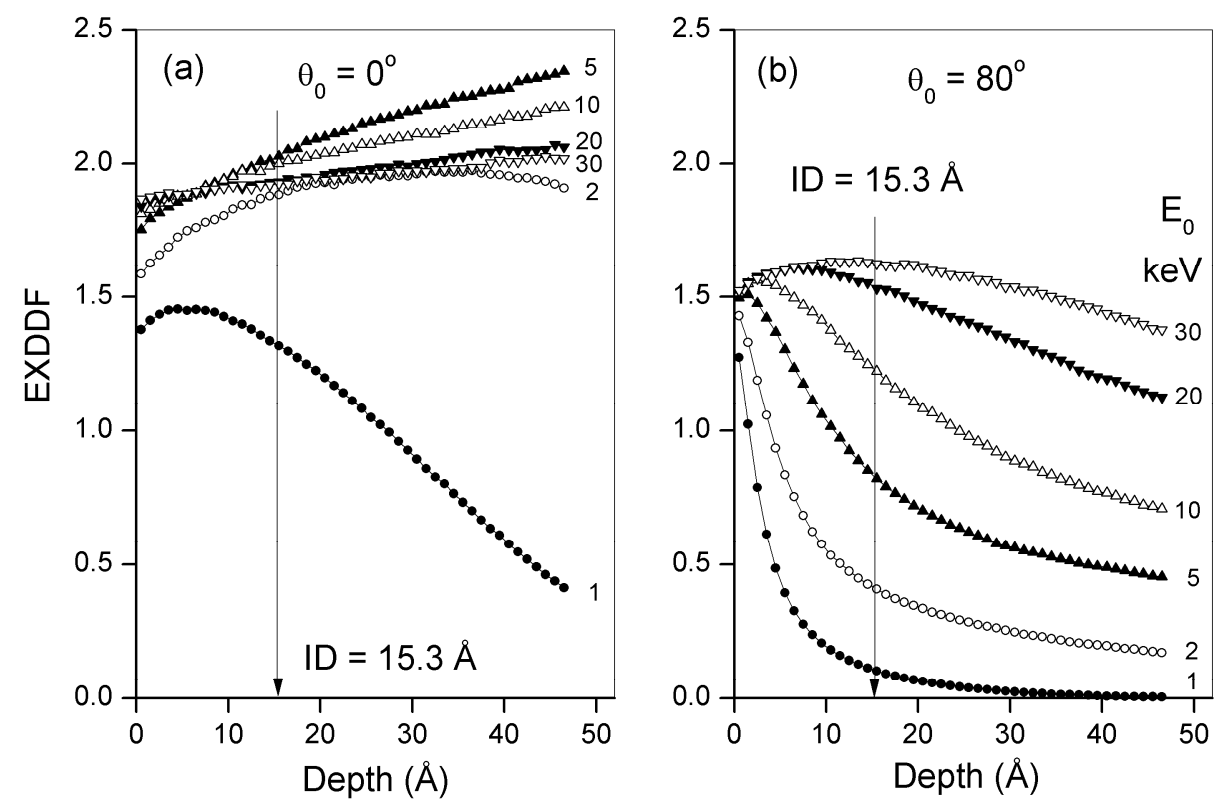

Fig. 3. The EXDDF calculated for the $\mathrm{Ag} \mathrm{M}_{4}$ subshell for the indicated primary energies between $1 \mathrm{keV}$ and $30 \mathrm{keV}$. Arrow indicates the $95 \%$ information depth. (a) Primary beam incidence angle $\theta_{0}=0^{\circ}$; (b) $\theta_{0}=80^{\circ}$. The vertical arrow indicates the maximum information depth (ID) for detection of $95 \%$ of the $\mathrm{Ag} \mathrm{M}_{4} \mathrm{~N}_{45} \mathrm{~N}_{45}$ Auger electrons.

normal to the surface in both of the considered cases, we see a distinct influence of the size of the solid angle on the IEMDDF. For comparison, the EMDDF calculated from the transport approximation [15], a more approximate model, for normal emission of the Auger electrons is also shown in Fig. 2. We see that this function compares well with the IEMDDF for an analyzer solid angle with $\Delta \alpha=10^{\circ}$. Thus, the IEMDDF does not appear to vary significantly for small half-cone angles, $\Delta \alpha$, less than $10^{\circ}$.

The second function needed for calculations of the BCF is the EXDDF [Eq. (3)]. This function calculated for the $\mathrm{Ag} \mathrm{M}_{4}$ subshell is shown in Fig. 3. The EXDDF clearly depends on primary energy and to a much greater extent for the more grazing incidence angle of the primary beam [Fig. 3(b)]. In the so-called common formalism of AES [1-3], the density of inner-shell ionizations is assumed to be uniform within the depth from which the detected Auger electrons originate. This assumption is also made in the simplified model for the BCF. The vertical lines in Fig. 3 indicate the maximum information depth (ID) for detection of $95 \%$ of emitted $\mathrm{Ag} \mathrm{M}_{4} \mathrm{~N}_{45} \mathrm{~N}_{45}$ Auger electrons. Figure 3 clearly shows distinct variations of the EXDDF within the ID, particularly for low primary energies and for glancing incidence of the primary beam. These variations lead to differences between the BCF values calculated from the advanced and simplified theoretical models.
Figure 4 compares the energy dependences of BCFs calculated from different theoretical models for incidence angles of (a) $0^{\circ}$ and (b) $80^{\circ}$. As expected, there are pronounced differences between BCFs calculated from the advanced and simplified models, particularly at glancing incidence of the primary beam. For a primary energy of $1 \mathrm{keV}$, the difference between BCFs from the simplified model and from the advanced model for $\theta_{0}=80^{\circ}$ and $\Delta \alpha=10^{\circ}$ exceeds $50 \%$. This difference decreases to $4.5 \%$ at $30 \mathrm{keV}$. At normal incidence, the difference is smaller, reaching $10 \%$ in the vicinity of $E_{0}=2 \mathrm{keV}$. A distinct difference is observed between $\mathrm{BCFs}$ calculated from the simplified model [Eq. (8)] and those from the Shimizu predictive formula [16]. The BCF difference varies between $10 \%$ and $13 \%$ for energies between $3 \mathrm{keV}$ and $10 \mathrm{keV}$ (the energy range for which the Shimizu formula was developed), and increases to $17 \%$ for higher energies. The Shimizu predictive formula is based on BCF calculations with the simplified model but different input parameters (elastic scattering cross sections, ionization cross sections, stopping powers) were used by Shimizu and coworkers [16-18] than in the present calculations [10,11]. In addition, there is a singularity at $\alpha_{B}=\pi / 2$ in Eq. (8), and this problem was circumvented in each algorithm in a different way. Finally, the Shimizu formula was obtained from fits to results 

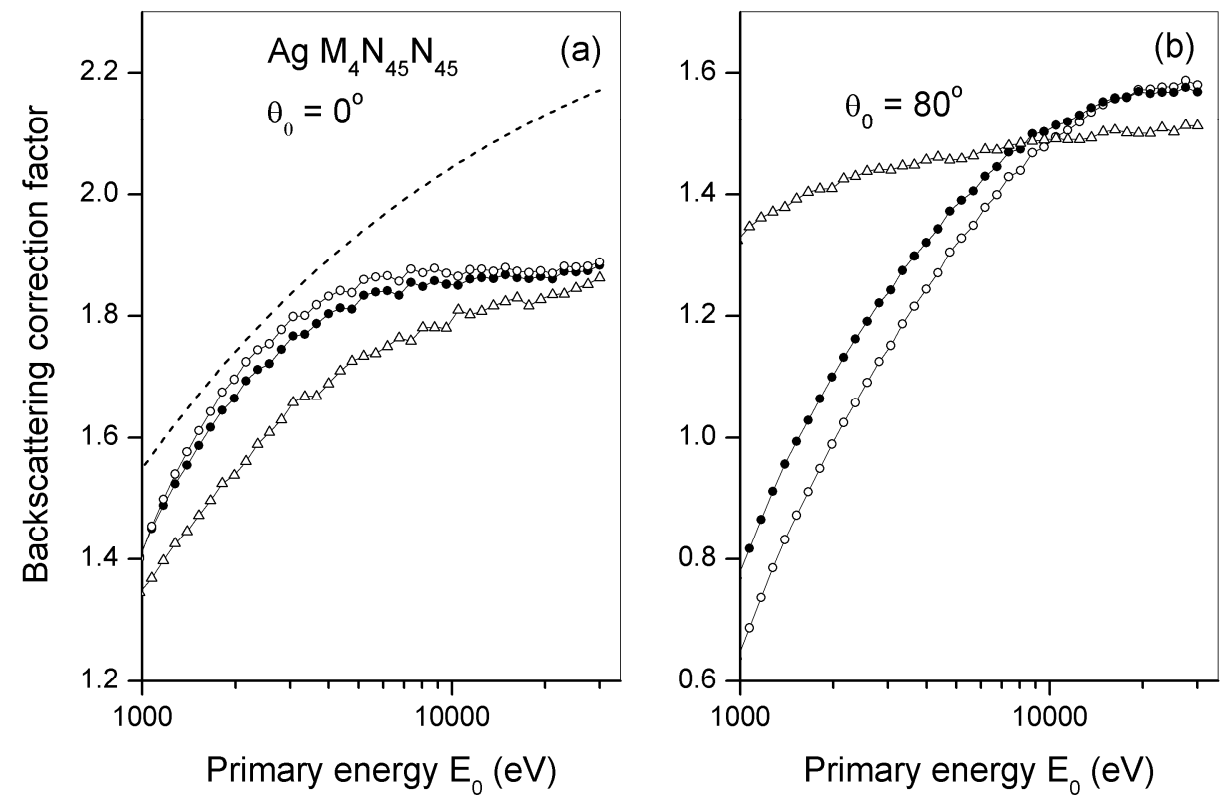

Fig. 4. Energy dependence of BCFs for $\mathrm{Ag}_{4} \mathrm{~N}_{45} \mathrm{~N}_{45}$ Auger electrons from silver. Open circles: advanced model for $\Delta \alpha=10^{\circ}$; filled circles: advanced model [Eq. (7)] for $\Delta \alpha=80^{\circ}$; triangles: simplified model [Eq. (8)]; dashed line: the Shimizu formula [16]. (a) Primary-beam incidence angle $\theta_{0}=0^{\circ}$; (b) $\theta_{0}=80^{\circ}$.

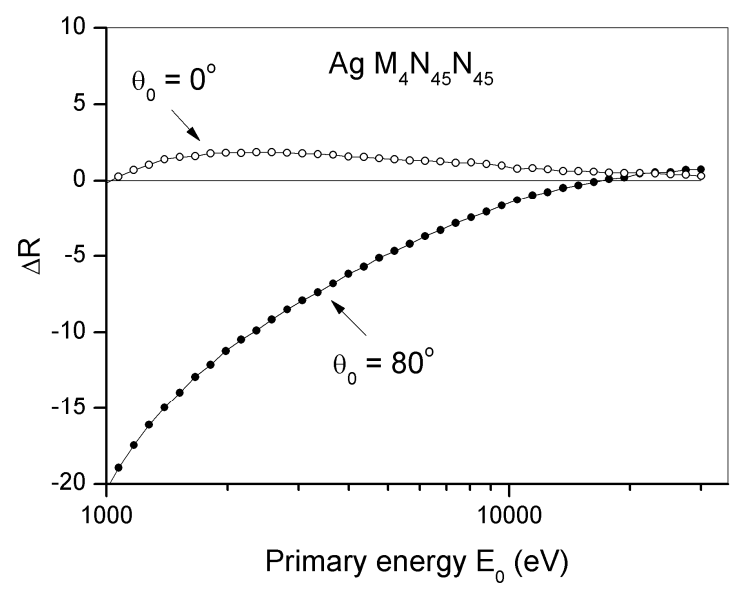

Fig. 5. Plot of the percentage difference $\Delta R$ from Eq. (9) between BCFs calculated from the advanced model for analyzer-acceptance solid angles with $\Delta \Omega=10^{\circ}$ and $\Delta \Omega=80^{\circ}$. The open circles show $\Delta R$ for a primary-beam incidence angle of $0^{\circ}$ and the solid circles show $\Delta R$ for an incidence angle of $80^{\circ}$.

of Monte Carlo calculations for many solids, and some differences of BCFs for individual solids from $\mathrm{BCFs}$ from the formula are expected.

We now calculate the differences between BCFs from the advanced model for the two considered solid angles, i.e., between $R\left(\Delta \alpha=10^{\circ}\right)$ and $R\left(\Delta \alpha=80^{\circ}\right)$ :

$$
\Delta R=100 \frac{R\left(\Delta \alpha=10^{\circ}\right)-R\left(\Delta \alpha=80^{\circ}\right)}{R\left(\Delta \alpha=10^{\circ}\right)} .
$$

Figure 5 shows plots of $\Delta R$ versus primary energy for incidence angles of $0^{\circ}$ and $80^{\circ}$. For normal incidence of the primary beam, $\Delta R$ is rather small, reaching $2 \%$ for primary energies between $2 \mathrm{keV}$ and $3 \mathrm{keV}$. Larger values of $\Delta R$ are observed for glancing incidence of the primary beam. At $1 \mathrm{keV}, \Delta R$ is about $20 \%$ but decreases with increasing energy to less than $1 \%$ at $30 \mathrm{keV}$. A similar result has recently been obtained for Pd $\mathrm{M}_{5} \mathrm{~N}_{45} \mathrm{~N}_{45}$ Auger electrons from palladium [11].

\section{The NIST BCF Database for AES}

Recent work has shown that BCFs from the advanced model are a function of the Auger-electron emission angle in addition to other parameters [6-11]. As shown above, the BCF also generally depends on the size of the analyzer-acceptance angle. We note that the solid acceptance angle of an analyzer may be complicated and require some additional defining parameters. Suppose that the solid acceptance angle is contained between two cones defined by half-cone angles $\Delta \alpha$ (external) and $\Delta \alpha_{i n}$ (internal), as outlined in Fig. 6 (e.g., as for a cylindrical mirror analyzer or a retarding-field analyzer with an axial electron gun). 
Furthermore, the analyzer axis may be located at a certain angle $\alpha_{a x}$ with respect to the surface normal. We see that three angles are then needed to describe the analyzer solid acceptance angle. It seems that it would be very difficult to develop a predictive formula for the $\mathrm{BCF}$ in which this angular information could be included. One of the possible solutions would be the availability of a computer-controlled database to provide BCFs from the advanced model for a user-specified experimental configuration. Such a database is currently under development [19]. The NIST Backscattering-Correction-Factor Database for Auger Electron Spectroscopy will provide BCFs from the simplified and advanced models for primary energies up to $30 \mathrm{keV}$. In calculations of the EXDDF, one of the three formulae for the inner-shell ionization cross section can be selected, i.e., the formulae of Gryzinski [20], Casnati et al. [21], or Bote et al. [22]. BCFs can be determined for ionizations of the K, L, M and $\mathrm{N}$ shells. For the advanced model, an experimental configuration as outlined in Fig. 6 can be selected. Finally, BCF data are available for elements and compounds.

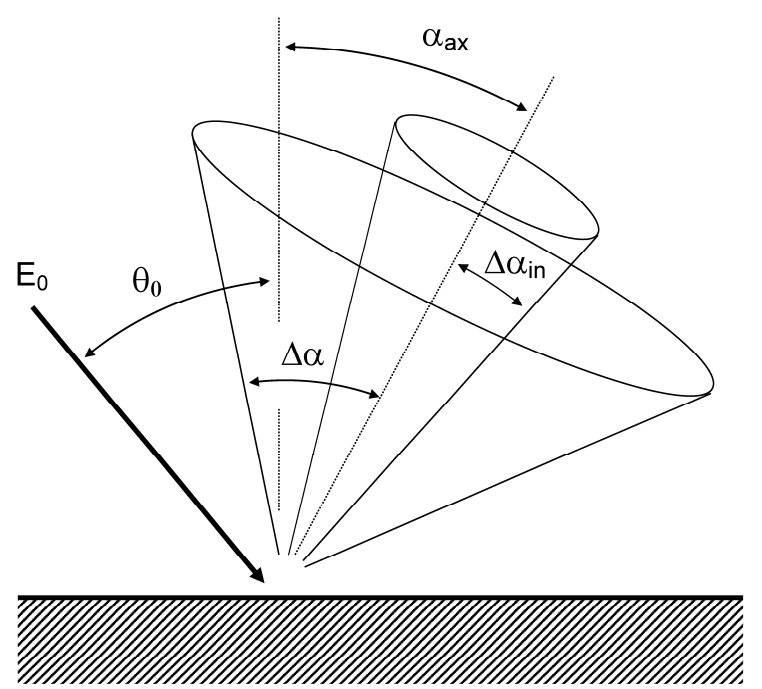

Fig. 6. An example of a complicated structure of the solid acceptance angle of an analyzer.

\section{Conclusions}

In view of advances in the theory of electron transport in solids, the definition of the correcting factor accounting in AES for ionizations by scattered electrons needs to be modified. The term "backscattering correction factor" is presently proposed for this correction. We find that the BCF depends on the size and shape of the analyzer acceptance angle. Preliminary calculations show that the influence of the analyzer-acceptance angle is not significant at normal incidence of the primary beam, but may be very pronounced at glancing incidence. Since numerous parameters influence the BCF, it would be difficult to derive a predictive formula for BCFs from the advanced model. A useful tool would be a database providing BCFs for particular analytical conditions [19].

\section{References}

[1] M. P. Seah and I. S. Gilmore, Surf. Interface Anal. 26, 723 (1998).

[2] M. P. Seah, J. Electron Spectrosc. Relat. Phenom. 100, 55 (1999).

[3] M. P. Seah, I. S. Gilmore and S. J. Spencer, Surf. Interface Anal. 31, 778 (2001).

[4] H. E. Bishop, J. C. Riviere, J. Appl. Phys. 40, 1740 (1969).

[5] A. Jablonski, Surf. Sci. 499, 219 (2002).

[6] A. Jablonski and C. J. Powell, Surf. Sci. 574, 219 (2005).

[7] Z. J. Ding, W. S. Tan, and Y. G. Li, J. Appl. Phys. 99, 084903 (2006).

[8] A. Jablonski and C. J. Powell, Surf. Sci. 601, 965 (2007).

[9] R. G. Zeng, Z. J. Ding, Y. G. Li, and S. F. Mao, J. Appl. Phys. 104, 114909 (2008).

[10] A. Jablonski, Surf. Sci. 603, 2047 (2009).

[11] A. Jablonski and C. J. Powell, Surf. Sci. 604, 1928 (2010).

[12] ASTM E 673-03, "Standard Terminology Relating to Surface Analysis" in: Annual Book of ASTM Standards, Vol. 3.06, ASTM International, West Conshohocken, 2009, p. 663.

[13] ISO 18115:2001, "Surface Chemical Analysis - Vocabulary", International Organization for Standardization, Geneva (2001).

[14] A. Jablonski and S. Tougaard, Surf. Sci. 432, 211 (1999).

[15] C. J. Powell and A. Jablonski, NIST Electron Effective-Attenuation-Length Database, SRD 82, Version 1.3 (2011); http://www.nist.gov/srd/nist82.cfm.

[16] R. Shimizu, Japanese J. Appl. Phys. 22, 1631 (1983).

[17] R. Shimizu and S. Ichimura, Quantitative Analysis by Auger Electron Spectroscopy, Toyota Foundation Research Report No. I-006 76-0175, Tokyo, 1981.

[18] S. Ichimura and R. Shimizu, Surf. Sci. 112, 386 (1981).

[19] A. Jablonski and C. J. Powell, NIST Backscattering-Correction-Factor Database 
for Auger Electron Spectroscopy, Standard Reference Data Program Database 154 (under development).

[20] M. Gryzinski, Phys. Rev. 138, A336 (1965).

[21] E. Casnati, A. Tartari and C. Baraldi, J. Phys. B: At. Mol. Phys. 15, 155 (1982); Erratum: J. Phys. B: At. Mol. Phys. 16, 505 (1983).

[22] D. Bote, F. Salvat, A. Jablonski, and C. J. Powell, At. Data Nucl. Data Tables 95, 871 (2009). 